\title{
外源添加营养对安徽太平湖水库 藻类增殖影响的研究
}

\author{
赵斌” \\ （水利部水库鱼业研究所.武汉 430073)
}

提要 1992 年 11 月 1993 年 10 月,在安微太平湖水库,同时用藻类生长潜力测试法 (AGP 试验) 和外源添加营养的黑白瓶测定初级生产力法,对陵山站的水体分季度进行了分析 试。其结果表明,这两种方法对太平湖水库的营养评价均是行之有效的,而且二者的结果也能 相互比较、相互验证; 太平湖水库的不同季节,其主要营养限制性因子也各异。在枯水期、磷是藻 类种群和密度的第一限制性暮养元素: 丰水期.由于各营养元素都相对缺乏,酸此时作为限制性 因子的作用下降。因而认为.在进行象太平湖水库这样的大水惐野外工作中,若谈同时运富这阿 种方法进行检验和比较分析.就能克服二者各自的片面性.得出轮为客观的结论。闹时、本实验 的结果对太平湖水库施肥养殖也有一定指导意义。

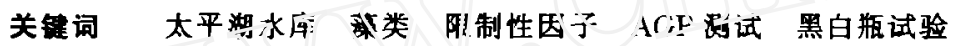

与地质姿动时期形成的湖泊相比,水库是一种相对年轻的淡水生态系统, 尚未进行广迄 的湖泊学或生态学研究 ${ }^{[1]}$ 。过去很多关于水库湖沼学的研究方法同湖泊学的研究方法基本 是一样的, 甚至认为水库和湖泊本质是相同的, 并将其划分成为第 73 类湖泊 ${ }^{[2]}$ 。但近年来的 研究表明, 人工建造的水库同天然湖泊并非必然相似的水体生态系统 ${ }^{[3 \sim 6]}$ 。它们的区别首先 在于物理环境要素 (包括水库的形态和水文学特性、区域性的地理因素以及流体力学因素) 的不同; 其次,在于生态系统内部营养结构、功能和动态上的差异, 其中, 浮游植物的营养结 构, 是反映生态系统营养结构的具体体现, 这是因为在绝大多数大中型水库中浮游植物占初 级生产力的主要优势。营养限制性因子是生态系统营养结构最重要的方面之一, 是影响水生 态系统中浮游植物季节性增长的重要因素, 同其它如捕食压力、光强或水体稳定性等因子相 比,要显得更为重要 ${ }^{[7]}$ 。水库中的浮游植物生活的环境在物理、化学和生物方面均是呈动态 变化的。虽然从年变化来看,水库中的浮游植物生产量的变化不如天然湖泊明显,但是如果 缩短时间期限进行比较, 水库中的变化可能要比天然湖泊大得多 ${ }^{[1]}$ 。本文试从太平湖水库中 浮游植物的营养限制性因子人手, 对外源添加氮、磷等营养物质进行动态研究, 监测其对藻 类增殖情况及水体初级生产力的影响,探讨浮游植物营养结构以及水库和湖泊生态系统的

：中国科学院"八五”宏观生物学重点项目 $(K Y 85-112)$ 资助。

** 现在工作单位:中国科学院水生生物研究所.邮政编码 430072 来稿日期: $1994-08-26$; 接受日期: $1994-10-23$ 。 作者简介: 赵陡.男.1969 年生.助理工程师。1992 年南京大学环境科学系环境生物学专业毕业。现主要从事汽水 生态学研究工作。 
区别, 旨在为水库和天然湖泊的比较生态学研究提供第一手资料, 同时也寻找一种研究水体 营养状况的野外操作方便的方法。

\section{1 材料和方法}

\section{1 试验水库概况}

太平湖水库位于安徽省著名旅游胜地黄山和九华山 2.8 当库容 $24.75 \times 10^{8} \mathrm{~m}^{3}$, 流域 面积 $2800 \mathrm{~km}^{2}$, 是一座峡谷型水库, 库长约 $80 \mathrm{~km}$, 可养殖水志河 $6600 \mathrm{hm}^{2}$, 几乎占整个水面 的 $80 \%$ 。库区属亚热带的过渡地带,年平均气温约 $16 \mathrm{C}$, 年太阳辐射能总量约为 $4.437 \mathrm{~K}$ $10^{3} \mathrm{~kJ} / \mathrm{cm}^{2}$ 。水位高程 $110 \mathrm{~m}$ 时面积为 $13.2 \times 10^{4} \mathrm{hm}^{2}$, 消落区 $3.3 \times 10^{4} \mathrm{hm}^{2}$, 最大泄洪量为 $4670 \mathrm{~m}^{3} / \mathrm{s}$, 库水流经陈村大坝人青式江后汇人长江。

\section{2 采样点的设置和水样预处理}

选择太平湖水库中游最具代表性的陵山站最开阔水面处采集水样。取 $1.0 \mathrm{~m} 、 2.0 \mathrm{~m}$ 、 $3.0 \mathrm{~m} 、 4.0 \mathrm{~m} 、 6.0 \mathrm{~m}$ 深的分层水样进行混合,用 $25^{ \pm}$生物网初过滤,再用 $0.45 \mu$ 的微孔滤膜过 滤，同时测定水样中氮、磷等元素的初始营养成份以及浮游藻类的种类、密度、生物量和叶绿 素a等指标 $($ 表 $1 、 2)$ 。

表 1 太平湖水库氮、磷等主要营养元素的季节变化 单位: $\mathrm{mg} / \mathrm{L}$

Tab. 1 Seasonal variations of nitrogen and phosphcuru: tte. i i Tapinghu Reservoir

\begin{tabular}{|c|c|c|c|c|c|c|c|}
\hline 营养元素 & T. & $T P$ & $\therefore U_{3}-N$ & $\mathrm{NO}_{\overline{3}}-\mathrm{V}$ & $\mathrm{NH}_{4}^{+}-\mathrm{N}$ & $\mathrm{PO}_{4}^{3-}-\mathrm{P}$ & $\mathrm{S}_{1} \mathrm{O}_{2}$ \\
\hline 营果(4.95) & $0.5 ? 1$ & 0.006 & 0.004 & 0.381 & 0.032 & $<0.01$ & 6. 92 \\
\hline 夏季(一 & 0.564 & 0.009 & 0.005 & 0.180 & 0.016 & $<0.01$ & 5.88 \\
\hline 秋季(10１1月) & 1.65 & 0.184 & 0.006 & 0.287 & 0.046 & $<0.01$ & 5.09 \\
\hline 冬季(1月) & 7.68 & 0.043 & 0.004 & 0.336 & 0.012 & $<0.01$ & 4.45 \\
\hline
\end{tabular}

表 2 太平湖水库浮游植物的季节变化

Tab. 2 Seasonal variations of properties of phytoplankton in Taipinghu Reservoir

\begin{tabular}{|c|c|c|c|c|c|}
\hline 季 & 节 & 类 & 密度 $\left(10^{3}\right.$ 个 $\left.L\right)$ & 生物量(mg L) & 叶绿素 $\mathrm{a}\left(\mathrm{mg} / \mathrm{m}^{3}\right)$ \\
\hline \multicolumn{2}{|c|}{ 春季(4月) } & 15 & 58.1 & 1.23 & - \\
\hline \multicolumn{2}{|c|}{ 夏季( 7 ～月 ) } & 17 & 37.2 & (1. 52 & 1.68 \\
\hline \multicolumn{2}{|c|}{ 秋季(10一 11 月) } & 21 & 35.4 & 1.113 & 1.79 \\
\hline \multicolumn{2}{|c|}{ 冬季(1 月) } & 23 & 88.5 & 1.68 & 3.47 \\
\hline
\end{tabular}

\section{3 试验方法}

为了使本研究结果同其他相关研究具可比性, 同时又能代表太平湖水库的浮游植物的 营养状况，试验选择了两种方法同步进行研究:(1) 藻类生长潜力 AGP 测试法 ${ }^{\circ}$ 。试验藻种 为由中国科学院水生生物研究所室内人工纯培养的斜生姗藻 (Scenedesmus obliquus)。(2) 外 源添加营养的黑白瓶试验

添加营养均为分析纯试剂。磷用磷酸二氢钠 $\left(\mathrm{NaH}_{2} \mathrm{PO} \mathrm{O}_{4} \cdot 2 \mathrm{H}_{2} \mathrm{O}\right)$ ), 氮用硝酸钾 $\left(\mathrm{KNO}_{3}\right)$ 。 使用前先用蒸馏水配制成浓度较高的母液.使用时再以培养用水稀释至所需浓度, 单独或共 同添加到培养用水中。 


\section{2 结果}

\subsection{AGP 证验}

为了探讨水库与湖泊不同的营养动态特性,试验特意安排在水库水位及营养状况变动 较大的时期 (4 10 月) 进行。检测太平湖水库浮游植物营养限制性因子的试验共进行 5 次: 1993 年 4 月 $\left(A_{1}\right) 、 1993$ 年 7 月 $\left(A_{2}\right) 、 1993$ 年 8 月 $\left(A_{3}\right) 、 1993$ 年 $9 \sim 10$ 月 $\left(A_{4}, A_{5}\right)$ 。实验结束 后, 求出最大特定生长率 $\left(\mu_{\max }\right)$ 和最大现存量等参数。

2.1.1枯水期，磷对萍类生长的限制性作用 太平湖水库一般在夏汛前水位降到最低点， 夏末秋初雨季后水位开始回升。1993 年 4 月,是当年水位最低的时期,处于枯水期。其添加 氮、磷营养后的生长动态见图 1( $\left(\mathrm{A}_{1}\right)$ : 添加磷的培养液同对照组相比, 有明显促进挪藻生长 的作用, 并且 $0.050 \mathrm{mg} \mathrm{P} / \mathrm{L}$ 组 $>0.015 \mathrm{mg} \mathrm{P} / \mathrm{L}$ 组。而同时氮的添加, 不仅不能促进梛藻的生 长, 甚至在某种程度上对藻类的生长还起一定的阻碍作用。这种机理尚需进一步探讨，

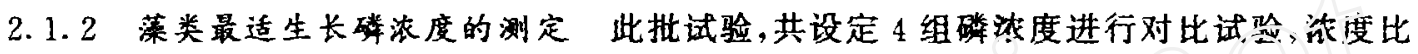

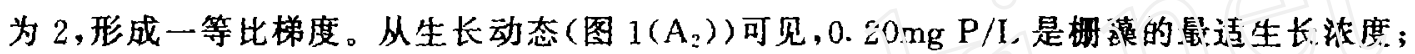

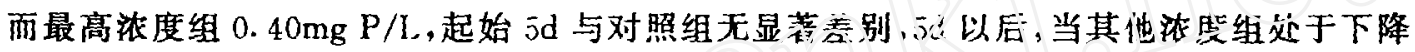

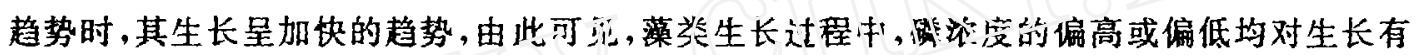
抑制作用。

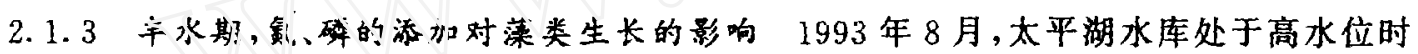
期。此期间汇集了一年中最丰富的降雨。该批试验中磷的浓度同上批试验浓度设置完全相 同,但结果却有较大差异。生长动态 (图 $1\left(\mathrm{~A}_{3}\right)$ ) 表明: 各组之间生长无显著差异, 此时期氮、 磷的添加不能显著地促进藻类增长。

为了进一步明确磷在丰水期的作用, 于 1993 年 9 月至 10 月重复了上批试验 ( $\mathrm{A}_{4}$, 图 1 $\left.\left(A_{4}\right)\right)$, 并在其它条件不变的情况下增加磷浓度重复此批试验 $\left(A_{5}\right.$, 图 $\left.1\left(A_{5}\right)\right)$, 结果表明, $A_{4}$ 和 $\mathrm{A}_{3}$ 的结论是一致的。在高浓度组的情况下, 随着磷浓度的增加, 䅘藻增长加快, 即梛藻生 长曲线呈现出“双峰型”。对此本文未作深人研究，有待今后详细探讨。

2.1 .4 其它影响营养因子的因素 为了确定水库中是否还存在着其它限制藻类生长的因 子, 利用两种落类培养基 $\mathrm{HB}_{4}$ 和 $\mathrm{T}_{5}$ 作了下述几组试验: 全库水、 $\mathrm{T}_{5}$ 培养基、库水 $+\mathrm{T}_{5}$ 培养 基、 $\mathrm{HB}_{4}$ 培养基、库水 $+\mathrm{HB}_{4}$ 培养基。其生长动态见 (图 $1\left(\mathrm{~A}_{6}\right)$ )。结果表明:库水水 $\mathrm{T}_{5}$ 培养基 组生长状态最为良好, 并且库水 $+T_{5}$ 培养基组 $>T_{5}$ 培养基组, 而库水 $+H B_{4}$ 组 $<T_{5}$ 组, $T_{5}$ 组 $>\mathrm{HB}_{4}$ 组。可以看出, $\mathrm{T}_{5}$ 营养基最适合于太平湖水库藻类生长, 可见太平湖水库中除了磷 是限制藻类生长的营养因素之外，还有很多复杂的营养关系。比较 $\mathrm{T}_{5}$ 培养基同 $\mathrm{HB}_{4}$ 培养基 成份的差别发现, $\mathrm{HB}_{4}$ 仅比 $\mathrm{T}_{5}$ 少 $\mathrm{NO}_{3}^{-} \mathrm{N}$, 但生长状况的差异却如此之大 (伡水 $+\mathrm{T}_{5}$ 培养基 组藻类最大现存量为 $9.92 \times 10^{6}$ 个 $/ \mathrm{ml}$, 而库水 $+\mathrm{HB}_{4}$ 组为 $5.54 \times 10^{6}$ 个 $/ \mathrm{mL}$ ), 因而 $\mathrm{NO}_{3}^{-}-\mathrm{N}$ 对于太平湖水库的藻类增殖起着举足轻重的作用。

\section{2 黑白瓶试验}

用于检测太平湖水库外源添加氮、磷营养对浮游植物初级生长力影响的黑白瓶挂瓶试 验共进行 3 次: 1993 年 1 月 $\left(\mathrm{B}_{1}\right), 1993$ 年 4 月 $\left(\mathrm{B}_{2}\right), 1993$ 年 7 月 $\left(\mathrm{B}_{3}\right)$ 。主要用于对 $\mathrm{AGP}$ 测试 中部分结论的验证, 实验与 AGP 测试同步进行, 以保证研究的代表性。其各次试验的总初 


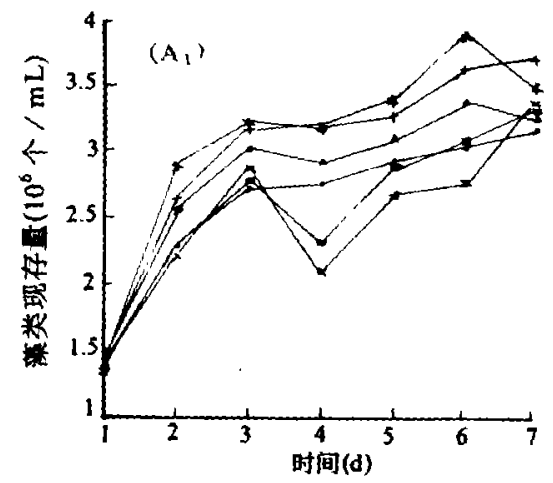

- 对盟的 $\quad++-0.015 \mathrm{mg} P / \mathrm{L}$

$\rightarrow 0.060 \mathrm{mgP} / \mathrm{L} \quad-0.225 \mathrm{mgN} / \mathrm{L}$

$-x-750 \mathrm{mgN} / \mathrm{L}: \quad-0.015 \mathrm{mgP} / \mathrm{L}+0.750 \mathrm{mgN} / \mathrm{L}$

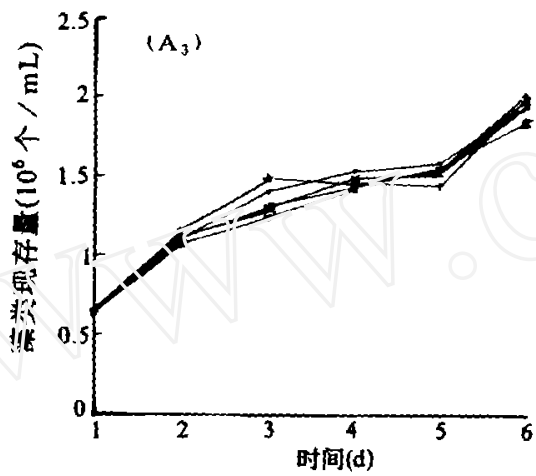

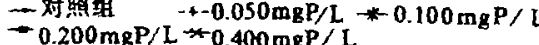

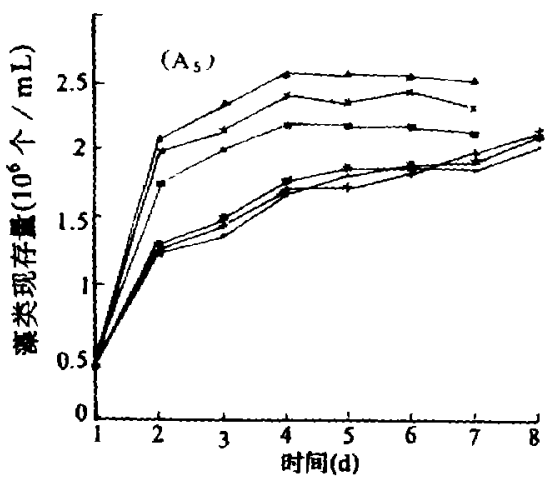

一对县组

$x \rightarrow 0.200 \mathrm{mg} P / L$

$-0.100 \mathrm{mg} P / \mathrm{L} \quad-x-0.050 \mathrm{mg} P / L$ $++0.025 \mathrm{mgP} / \mathrm{L} \quad--0.400 \mathrm{mg} / \mathrm{L}$

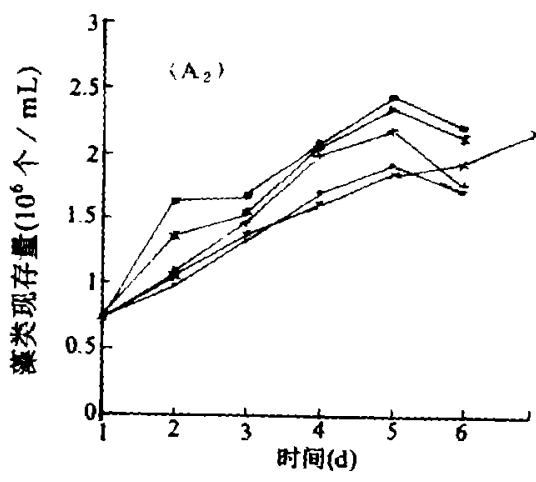

$$
\text { - 对盟组 } \quad-+-0.050 \mathrm{mgP} / \mathrm{L}
$$
$-*-0.100 \mathrm{mg} P / L$

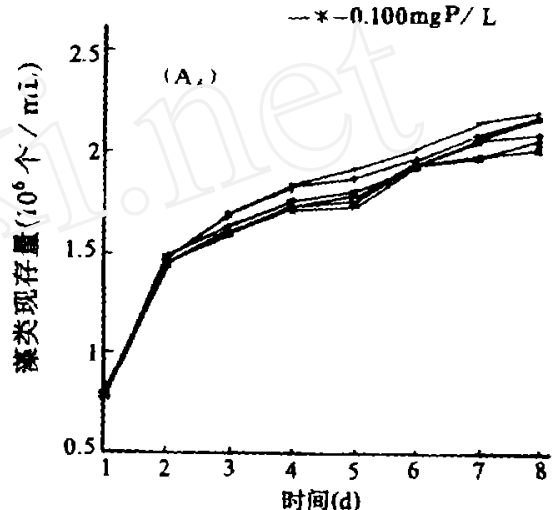

- 对然组 $\quad-x-0.200 \mathrm{mg} P / I$ $\rightarrow 0.100 \mathrm{mg} P / \mathrm{L} \rightarrow-0.050 \mathrm{mg} / \mathrm{P} / \mathrm{L}$ $-4-0.025 \mathrm{mg} \mathrm{P} / \mathrm{L} \rightarrow-0.400 \mathrm{mg} / \mathrm{L}$

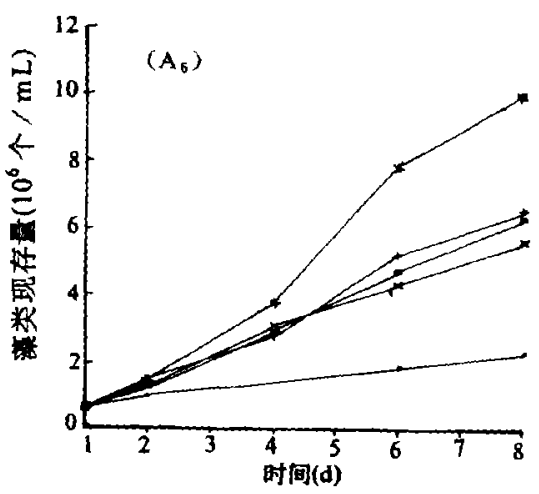

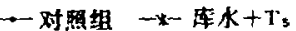

$+T_{s} \rightarrow H B$

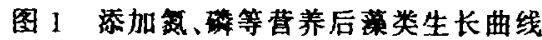

Fig. 1 Algal growth curves after adding $N$ and $P$ 

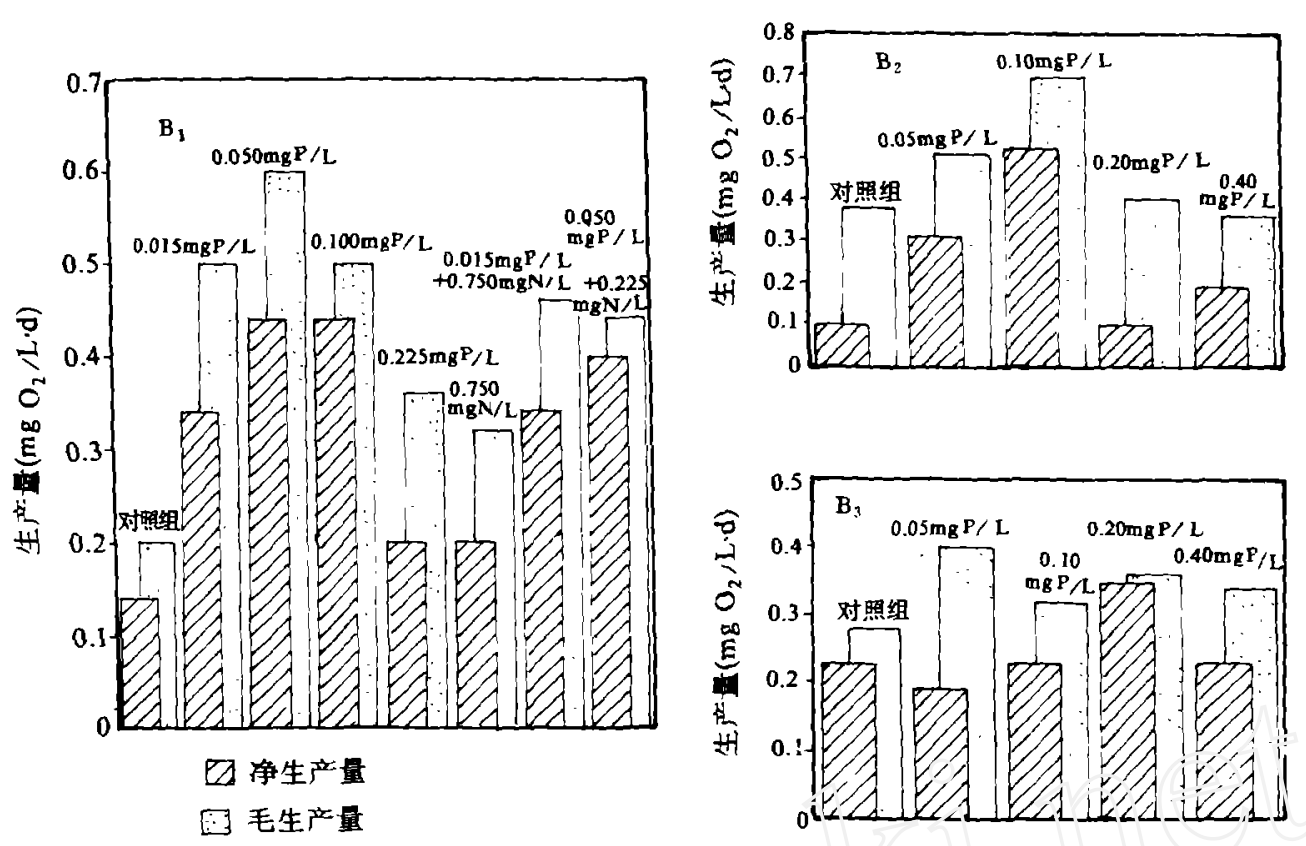

图 2 总苆级生产量和净狠级生王量的比较

$F_{i g}$ \& The cen:parison of gross pruduction and net production

级生产力和等邚纱生产力见图 2 。

2.2 .1 氛、磷营养元素的添加对萍类初级生产力的影响 $\mathrm{B}_{1}$ 试验共分 8 组进行,各组设定 不同的氮、磷浓度或配比。其结果表明, 磷在 $0 \sim 0.100 \mathrm{mg} / \mathrm{L}$ 浓度范围内, 净初级生产力随浓 度升高而增大, 毛生产力也基本呈现这一趋势; 而加氮组藻类初级生产力却无明显随氮浓度 升高而增加的现象，但同对照组相比，氮的添加对生长也有一定的促进作用。氮、磷混合组也 基本是依赖磷浓度的升高而变化的。因而, 在此时期, 磷对藻类初级生产力相对于筑更重要, 进一步证明磷是藻类生长的营养限制性因子。

2.2 .2 促进萍类初级生产力的最适磷浓度从 $\mathrm{B}_{2}$ 结果可以明显地看出藻类的初级生产力 随磷浓度增大而增加的情况。 $0.10 \mathrm{mgP} / \mathrm{L}$ ，对此时期的太平湖来讲，是藻类最适生长浓度。

2.2 .3 丰水期，的添加对落类初级生产力影响不大 $B_{3}$ 试验各组同对照组相比，均在对 照组附近波动, 差异不显著，可见在丰水期磷的添加对藻类初级生产力的促进作用不明显。

\section{3 讨论}

\section{1 对本试验中所应用的方法的比较评价}

在此选用了两种广泛应用于湖沼学上的方法对太平湖水库营养限制性因子进行了探 讨。这两种方法都是外加营养源生物测试法, 试验周期短, 它们是判定水体限制性营养元素 和预测水体营养状况的通用方法, 这些方法能直接查明水体中营养盐对浮游植物的供应情 况，包括各种营养元素的相对丰度和限制性营养元素的种类等。以往的许多学者往往比较推

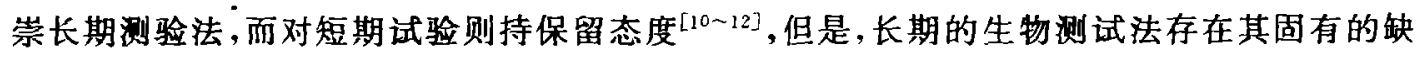


淊：(1) 这些方法一般需要一周至数周时间或更长时间,时间太长,培养液中的生物的组成 会发生同研究水体许多完全不同的变化 ${ }^{[13.14]}$; (2) 时间太长,培养液中营养状况会发生同研 究水域不相同的变化 (例如限制性营养因素的改变及营养物可利用性的改变 ${ }^{[10.14]} ;(3)$ 时 间太长,其试验结果用来指导不断变化的水体的施肥实践将有较大出人。短期试验相对地克 服了这些缺点,提高了它在生产实践中的应用价值。

比较分析所选用的两种方法,可以发现,不管从形式上还是所提供的结果,它们都是确 切的、有意义的,且试验设备简单,试验过程容易控制, 两种方法得到的结论也基本一致,这 无疑使二者独立的结果能够相互借证，从两种不同形式的方法得出了同一结论，应当相信其 结论的可靠性。而且, AGP 测试还是国内外进行生物刺激(藻类生产力)实验的标准方法.已 有很多的相关研究,应用这种方法所得出的结果可便于进行参照和比较; 本文中的外源添加 营养的黑白瓶方法虽不是标准方法,但它却是参照标准方法进行的,同样能提供较为可靠的 结论。因而作者认为, 对于进行象太平湖水库这样的大水域野外研究工作,这两种方法是可 取的。上述两种方法在对藻类生长最适磷浓度的测定上略有差异,这可能主要是因为二者在 藻种及培养时间等的差异所造成。诚然,这两种方法各自也存在一定的缺陷: AGP 试验中所 接种的斜生柟落不能完全代表水库所有的浮游植物，因为不同种类的雚类对常养源的配比 有不同的要求 ${ }^{225}$, 因而如果仅以此来评价水体的营养状况,显然存在较大出人。㫮已瓶试验 利用了水库中本身浮游植物群落进行测定，克的丁上过弱点，对于反映了示库浮游植物的实

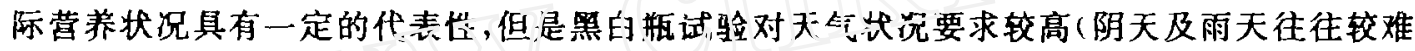

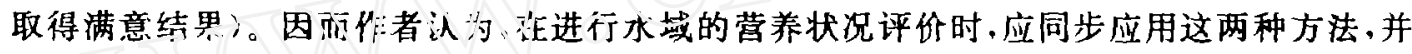
对实验结梁让行红致的比较分析, 寻找造成结果差异的原因,才能得出较为可靠的结论, 正 确地指导渔业生产实践。

\section{2 水库磷、虫限制作用的变化在施肥养殖中的应用}

水库一般具有多方面的功能。太平湖水库是一座集发电、灌溉、航运、养殖以及旅游等多 功能水体。水库和天然湖泊在排水方式上是不同的，水位的日变化和季节生波动程度也明显 不同,天然湖泊在表层排水,排水不可控制, 但水位很少发生剧烈变化,而水库却因改变或控 制河川流量的蓄水、灌溉过程引起的水位的急剧变化，同时水库还会因水力发电排放的不同 水温的水所引起水温变化,这些将导致发生在水库和其他类型的水体中的营养状况及其相 关的过程具有明显的不同。水伡的水质和生产力在很大程度上受着输人的外源性营养物的 质和量的控制，因而对水库营养状况的探讨应以动态研究为佳。本研究结果表明: 太平湖水 库在枯水期，磷对水体中初级生产力的限制作用比氮重要，磷在此时是藻类种群和密度的第 一限制性营养元素。而且，此时的䔀类最适生长的磷浓度为 $0.10 \sim 0.20 \mathrm{mg} / \mathrm{L}$ 之间, 此结果 对水库施肥养殖应有参考价值。结合表 1, 夏、秋雨季的降水导致水库中 $\mathrm{NO}_{3}^{-}-\mathrm{N}^{-}$农度降低,

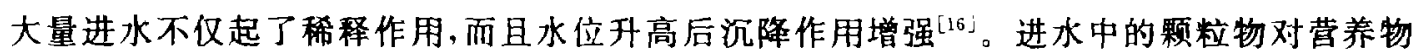
质的吸附作用使得水库中的 TP、TN 浓度及其他一些营养物质的浓度均不同程度地降低, 但氮元素浓度的降低并未达到限制浮游植物生长的程度,因而还不可能成为藻类生长的限 制性因素。结合表 2 清惭可见, 在夏、秋两季,无论是浮游植物的密度、生物量,还是叶绿素 a 的含量,均低于春、冬两季。夏、秋两季的 TP 浓度虽有增无减,但生物可直接利用的磷,例如 $\mathrm{P}()_{4}^{3-}-\mathrm{P}$ 浓度却并未增加, 即使人为添加磷也并未促进藻类增长。这表明: 磷在此时作为限制 
性因子的作用下降, 同时由于其它许多营养元素因雨水的稀释和吸附作用使浓度降低, 也相 对缺乏，因而磷、氮的添加在此时不能显著的促进藻类的增长及水体初级生产力的提高是容 易理解的。这提示在水库增肥同时应配和氮磷元素添加多种肥料。对照完全培养基的试验 还说明，太平湖水库中除了氮、磷之外，其它一些营养元素的缺乏构成了抑制㩰类存在或生 长教殖的综合因素。

显然,对太平湖水库的营养状况的评价，不能一概而论，也不能仅仅依靠一、两次营养评 价实验下结论，而应当针对不同时期、不同水文环境进行评价，不同时期的施肥方案也相对 有所改变。同天然湖泊相比,水库因受气象和水文学因素的变动影响而导致其生产力状况经 常性的扰动,往往表现出更大的变化速率。但是由于水库水中停留时间较短和外来营养物的 输人，而表现出其生态系统的动态稳定性和回复力,使其年观测数据表现出较大的重复性。 因而对水库的营养状况评讦应以一年为好,若能缩短时间间隔，将更为准确些。

致谢本研究工作是在夏宜璉教授、况琪军工程师的指导下进行的，其中水化学数据由 李植生提供，浮游植物数据由况琪军提供。参加野外采集和室内测定工作的还有总志文。在

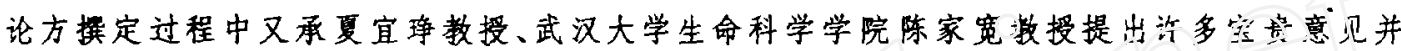
审改全文,谨此京心感谢。

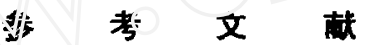

1 Kent W T. Perpertres on reservoir limnology. In: Kent W Thornton, et al eds. Reservoir limnology : ecological perspecuves. New York: John Wiley \& Sons Inc, 1990. 1 13

2 Hutchinson G E. A treatise on limnology, Vol 1: Geography, physics, and chemistry. New York: John Wiley \& Sons Inc, 1957.1015

3 Baxter R M. Environmental effects of reservoirs. In:D Gunnison, ed. Microbial processes in reservoirs. Boston: Dr W Junk Publishers, 1985. I 26

4 Baxter R M. Environmental effects of dams and impoundments. Ann Rev Ecol and Syst, 1977, 8:225 283

5 Kennedy R H. et al. Characterization of the reservoir ecosystem. In: Gunnison D, ed. Microbial processes in reservoirs. Boston: Dr W Junk Publishers. 1985. 27 38

6 Thornton K M, et al. Reservair sedimentation and water quality-A heuristic model. In: Stefen H G. ed. Proceedings of the symposium on surface water impoundments. Amer Soc Civil Engr, New York, 1981. 654 661

7 Nedoma J. A seasonal study of phosphorus deficiency in a eutrophic reservoir. Freshwater Biology, 1993, 30(3):369 376

8 USEPA. Algal assay procedure: bottie test. orvallis. Ore: USEPA. National Environ Res Center. 1971. $1 \sim 82$

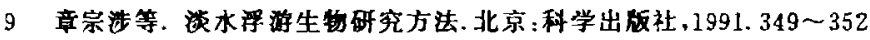

10 Fitzgerald G P. Bioassay analysis of nutrient availability. In: Allen and Kramer, eds. Nutrient in natural waters. 1972. $147 \sim 170$

11 Gerhart D Z, et al. Enrichment experiments for determining nutrient limitation; four methods compared. Limnol $O$ ceanogr. 1975. 20:640 653

I2 Lean D R S. et al. Photosynthetic response of lake plankton to combined nitrogen enrichment. J Phycd. 1982. 18(4): $509 \sim 521$

13 Kalf J. Nutrient limiting factors in an Arctic tundra pond. Ecology. 1971. 52:655 659

14 O'Brain W J. et al. Response of three phytoplankton bioassay techniques in experimental ponds of known limiting nutri- 
ent. Hydrodiod. 1976. 49(1):65 76

15 Smith V H. The mutrogen and phosphorous dependence of algal biomass in lakes: an empirical and theoretic analysis. Limnol Oceanogr . 1982. 27(6):1101 1112

16 李篦尚、浅水水库中的周年变动及其影响因素、海洋与湖沼.1991.22(2):104 110

\title{
STUDIES ON INFLUENCE OF EXOGENOUS NUTRIENT ON ALGAL GROWTH IN TAIPINGHU RESERVOIR
}

Zhao Bin

(Institute of Reserionr Fusheries. Minustry of Water Conservaricy \& Chinese Academy of Sciences. Wuhan 430073)

\begin{abstract}
The influence of exogenous nutrients on alga! growth was studiet in Lingshan Station of Taipinghu Reservoir, Anhui Province during the algal growth season of $1992 \sim 1993$. Two short-term algal bioassays, i. e. , a! gai growth potential ( $\triangle$ C.P'; test and black-light bottle test-the main experimentai method of determining nutrient limitation for phytoplankton and graen: macroaizate--were performed integratedly in various seasons in the site experiments. The main: additive used included phosphorus $\left(\mathrm{NaH}_{2} \mathrm{PO}_{4}\right)$ and nitrogen $\left(\mathrm{KNO}_{3}\right)$. The algae was Scenedesmus obliqus.

According to these experiments, the results show:

(1) The two methods were effective to reveal the trophic state of Taipinghu Reservoir and the results can be extended to large and medium reservoirs in the Changjiang River Basin. The respective one-side defect could be avoided and the two methods be used integratedly.

(2) The nutrient limitation of algae varied widely during the study period. In early summer, only the addition of $P$ evoked a clear increase in the growth of algae, revealing that $P$ was the primary limiting nutrient of algal population, biomass and primary production. In late summer and autumn, neither the addition of $\mathrm{P}$ nor the addition of $\mathrm{N}$ stimulated algal growth as in early summer because of the relative deficiency of other nutrition by the dilution of rainwater in summer rainy season, and the limiting action of $P$ was declining.

(3) The optimum $\mathrm{P}$ concentration for $S$. obliqus growth was $0.20 \mathrm{mg} / \mathrm{L}$.

The aforementioned analysis can be used for guiding technique of artificial fertilization in large water bodies. Many nutritions should be supplied when $\mathrm{P}$ and $\mathrm{N}$ are added in late summer and autumn in the Reservoir.
\end{abstract}

Key Words Taipinghu Reservoir, algae, nutrient limitation, algal growth potential test, black-light bottle test 